\title{
From traditional to whole-genome sequencing biomarkers for gastric cancer
}

\author{
"...personalized treatment of gastric cancer is \\ still in its infancy.
}

\begin{abstract}
Keywords: biomarkers $\bullet$ gastric cancer $\bullet$ next-generation sequencing analyses $\bullet$ personalized medicine $\bullet$ regulatory networks $\bullet$ tumor heterogeneity $\bullet$ whole-exome sequencing

- whole-genome sequencing
\end{abstract}

Despite advances in traditional research and identification of important single genes such as $C D H 1$, personalized treatment of gastric cancer is still in its infancy. In this editorial, we summarize the potential and challenges of comprehensive high-throughput analyses, including whole-genome sequencing (WGS) and microarrays-based gene expression profiling in clinical samples. Such a revolutionary strategy can lead to the discovery of nextgeneration biomarkers for gastric cancer.

Gastric cancer remains a major health problem, despite its declined incidence [1]. This type of cancer is associated with high mortality rates, particularly in advanced stages [1]. Understanding the genetic bases of this enigmatic type of cancer will offer novel insights into its pathogenesis and help identify new biomarkers. Recently, next-generation sequencing (NGS) applications have become a powerful and revolutionary tool for cancer research, and NGS studies have been also performed in gastric cancer samples [2].

\section{Advances in gastric cancer biomarkers}

The potential of endoscopic screening to detect high rate of early gastric cancer in East Asia has led to the establishment of endoscopic mucosal resection (EMR) and endoscopic submucosal dissection in the treatment of selected patients with early gastric cancer [3]. According to the current guidelines, EMR is recommended for patients with Tis or T1a tumors [4]. After endoscopic resection, examination of depth of tumor inva- sion, cancer differentiation and lymphatic involvement allows the prediction of the risk of lymph node metastases [3]. Currently, the indications for EMR include intestinal type adenocarcinoma; tumor with less than $2 \mathrm{~cm}$ diameter; without ulceration within tumor; and no lymphatic and vascular involvement. Recently, endoscopic submucosal dissection technique was introduced in order to extend the ability of EMR to remove lesions $>2 \mathrm{~cm}$, as EMR is limited only to the resection of small tumors [3,5]. These endoscopic approaches have dramatically improved quality of life by preventing open or laparoscopic gastrectomy.

Moreover, surgery is recommended for T1b-2 stage or higher and any $\mathrm{N}$ stage, but without distant metastases (M1) [6]. Adequate (R0) gastrectomy is recommended for $\mathrm{T} 1 \mathrm{~b}-3$ tumors, while T4 tumors require resection of involved structures. Extended (D2) lymph node dissection has been widely known as the gold standard for treatment of curable gastric cancer in eastern countries in the last decades. Contrarily, in western countries, D2 only recently became a recommended surgical approach [6]. Systemic chemotherapy has been standardized in both the West and the East, although there are some differences in the cytotoxic regimens $[4,6-8]$. In the metastatic setting, standardized systemic chemotherapy leads to overall improved survival to approximately 12 months $[4,8,9]$.

Moreover, traditional and new genetic tools have improved the effort toward personalized treatment of gastric cancer.

\section{Georgios D Lianos}

Centre for Biosystems \& Genomic Network Medicine, University of Ioannina, Ioannina, 451 10, Greece and

Department of Surgery, Ioannina University Hospital, loannina, Greece

\section{Christina D Bali}

Department of Surgery, Ioannina University Hospital, Ioannina, Greece

\section{Christos Katsios}

Department of Surgery, Ioannina

University Hospital, loannina, Greece

\section{Dimitrios H Roukos}

Author for correspondence: Centre for Biosystems \& Genomic Network Medicine, University of loannina, Ioannina, 451 10, Greece and

Department of Surgery, Ioannina University Hospital, Ioannina, Greece and

Biomedical Research Foundation of the Academy of Athens, Athens, Greece Tel.: +302651007423 Fax: +302651007094 droukos@uoi.gr 
Important traditional prognostic factors have been established from several clinical studies for gastric cancer [10,11]. Depth of tumor invasion, lymph node involvement and presence of distant metastases, all represent traditionally established prognostic factors essential for therapeutic decision-making for patients with gastric cancer [10-12]. There has been controversy about the prognostic significance of BMI [11,13], but the number of metastatic lymph nodes and the lymph node ratio have both been considered the most important prognostic factors in patients with gastric cancer by the American Joint Committee on Cancer in recent studies $[11,12]$. Interestingly, lymph node ratio has been proposed as a robust predictor of survival and it seems that it has a crucial prognostic role in patients with gastric cancer [11].

\section{Genetic factors}

Cancer genetics has led to the discovery of two important genes in routine clinical practice. In 1998, CDH1 was discovered and has recently been used for decisionmaking in the prevention setting. In the treatment setting, Her2 amplifications determine treatment with trastuzumab.

\section{"...traditional and new genetic tools have improved the effort toward personalized treatment of gastric cancer."}

It is clear that primary prevention is a major goal to reduce mortality. Toward this, genetic testing in individuals with a family history of diffuse gastric cancer permits the identification of family members with mutations at $\mathrm{CDH} 1[14,15]$. These mutation carriers have a very high risk of developing diffuse gastric cancer, and women with these mutations have an additional breast cancer risk (lobular type) [14]. In addition, the E-cadherin protein encoded by $\mathrm{CDH} 1$ is important in the molecular connections between adjacent cells in the stomach and breast. Recent evidence suggests that individuals with E-cadherin mutations have a lifetime risk of about $80 \%$ of developing diffuse gastric cancer and women with this mutation have an additional $40 \%$ risk of developing breast cancer (lobular type). For these reasons, we have to highlight that the scientific community has already established that prophylactic total gastrectomy should be performed for CDH1 mutation carriers [14,16].

In the therapeutic setting, recent insights in molecular-targeted therapeutic approaches, including Her2, represent a big hope in the therapeutic armamentarium against gastric cancer [17]. Application of Her2-targeted therapies, in particular, trastuzumab in combination with chemotherapy in patients with metastatic Her2-positive gastric cancers, has resulted in important improvement of overall survival rates [17]. Interestingly, trastuzumab is a fully humanized monoclonal antibody that binds to the extracellular domain of the receptor, acting by blockage of the Her2 receptor in addition to inducing antibody-dependent cellular cytotoxicity.

We have to highlight that, as with breast cancer, many patients with gastric cancer develop therapeutic resistance to trastuzumab. Furthermore, a substantial fraction of these patients, who account for $25 \%$ of all patients, develop recurrence, while for the remaining $75 \%$, no targeted therapy exists $[17,18]$.

Therefore, several promising agents are currently being developed in combination with chemotherapy to increase efficacy and overcome therapeutic resistance. Nowadays, an alternative is offered by the agent trastuzumab emtansine (T-DM1). T-DM1 provides increased therapeutic efficacy in Her2-positive breast cancer patients and we hope that similar encouraging results will be achieved in Her2-positive gastric cancer patients in the near future $[18,19]$.

It is clear that sequencing the gastric cancer genome has opened a new roadmap in the discovery of clinically important biomarkers in gastric cancer.

\section{Next-generation biomarkers}

The advent of NGS technology has undoubtedly revolutionized biomedical research [2]. These technologies have also recently been performed in gastric cancer genomics, allowing the assessment of gastric cancer heterogeneity and complexity. NGS studies include the sequencing of protein-coding genes (exome), termed as whole-exome sequencing (WES), in addition to both exome sequencing (1.5\% of the genome) and the noncoding part of the genome $(98.5 \%)$, termed WGS. These WES/WGS studies can identify genetic and genomic heterogeneity in clinical samples from patients with gastric cancer [2].

To date, two WES and two WGS studies have been published on gastric cancer. In 2011, Wang and colleagues published for the first time a WES study on 22 gastric cancer patients [20]. On the bases of WES analyses of 22 cancer-normal tissue pairs, they confirmed 19 previously identified driver genes and reported the detection of one significantly mutated gene (ARIDIA), a key member of the SWI-SNF complex. The somatic mutation rate of ARIDIA varies significantly among different subtypes of gastric cancer. Wang and colleagues have reported that the mutated ARIDIA gene was a prognostic factor independent of tumor stage [20].

In the second WES study, Zang et al. confirmed ARIDIA as a gastric cancer driver gene in 15 tumornormal tissue pairs analyses of gastric cancer [21]. Notably, a new driver gene (FAT4), a member of the E-cad- 
herin family, with a crucial role as a tumor suppressor gene was identified by Zang et al.

Two WGS studies in gastric cancer patients were published last year. A WGS study in 49 cancer-normal tissue pairs was reported by Wong SS et al. [22]. A total of 31 samples with diffuse and 18 with intestinal histological subtypes respectively were included in this study. Wong SS. et al. described frequent mutations in Ephrins and SLIT/ROBO signaling pathway genes [22]. There was heterogeneity in the mutational landscape between intestinal-type and diffuse-type gastric cancer [22].

\section{"Our major hope is that the NGS-based scientific era will open critical crossroads toward real genome-guided patient stratification and true personalized cancer medicine.}

One of the largest WGS studies for gastric cancer, and any cancer type, was reported by Wang et al. on 100 gastric cancer patients [23]. Aside from 100 tumornormal tissue pairs WGS analyses, gene expression and methylation profiling was carried out using microarray analyses. Wang et al. confirmed previously identified gastric cancer genes such as TP53, ARIDIA and $C D H 1$ [23]. Notably, four novel significantly mutated genes (UC6, CTNNA2, GLI3 and RNF43) were identified. Moreover, they identified $R H O A$ mutations in approximately $14.5 \%$ of diffuse-type tumors, while no such gene was identified in intestinal-type gastric cancers. These findings reveal and highlight the genomic complexity and heterogeneity of this type of cancer [23].

\section{Future perspective}

These NGS-based genomic studies prove the power of these technologies to identify new gastric cancer driver genes.

The RHOA mutation identified by the larger WGS study, which is uncommon for other cancer types, highlights the crucial role of this gene in driving diffuse-type gastric carcinogenesis [23]. This discovery of the RHOA pathway shapes a new horizon in the discovery of future therapeutics, although, transcriptional regulation and functional genomic studies will be necessary for gastric cancer management.

\section{Conclusion}

Our major hope is that the NGS-based scientific era will open critical crossroads toward real genomeguided patient stratification and true personalized cancer medicine [2,20-23]. Because, gastric cancer is a highly heterogeneous disease, many more WES and WGS studies with much larger sample sizes will be required in the future to establish the list of gastric cancer driver genes. Such a reference database creates a new environment for the discovery of biomarkers, to aid tailoring of the best treatment to the right patient at the right time.

\section{Financial \& competing interests disclosure}

The authors have no relevant affiliations or financial involvement with any organization or entity with a financial interest in or financial conflict with the subject matter or materials discussed in the manuscript. This includes employment, consultancies, honoraria, stock ownership or options, expert testimony, grants or patents received or pending, or royalties.

No writing assistance was utilized in the production of this manuscript.

\section{References}

1 Lianos GD, Rausei S, Ruspi L et al. Laparoscopic gastrectomy for gastric cancer: current evidences. Int. J. Surg. 12(12), 1369-1373 (2014).

2 Watson IR, Takahashi K, Futreal PA, Chin L. Emerging patterns of somatic mutations in cancer. Nat. Rev. Genet. 14(10), 703-718 (2013).

3 Uedo N, Takeuchi Y, Ishihara R. Endoscopic management of early gastric cancer: endoscopic mucosal resection or endoscopic submucosal dissection: data from a Japanese high-volume center and literature review. Ann. Gastroenterol. 25(4), 281-290 (2012).

4 NCCN (National Comprehensive Cancer Network). National Comprehensive Cancer Network Guidelines 2012. Gastric cancer. www.nccn.org

5 Gotoda T, Kusano C, Moriyasu F. Future perspective of gastric cancer endotherapy. Ann. Transl. Med. 2(3), 25 (2014).

6 Bali CD, Lianos GD, Roukos DH. Gastric cancer guidelines and genome differences between Japan and the west. Future Oncol. 9(8), 1053-1056 (2013).

7 Bang Y-J, Kim Y-W, Yang H-K et al. Adjuvant capecitabine and oxaliplatin for gastric cancer after D2 gastrectomy (classic): a Phase 3 open-label randomised controlled trial. Lancet 379 (9813), 315-321 (2012).

8 Sakuramoto S, Sasako M, Yamaguchi T et al. Adjuvant chemotherapy for gastric cancer with S-1, an oral fluoropyrimidine. N. Engl. J. Med. 357(18), 1810-1820 (2007).

9 Rolfo C, Bronte G, Sortino G et al. The role of targeted therapy for gastrointestinal tumors. Expert Rev. Gastroenterol. Hepatol. 8(8), 875-885 (2014).

10 Yasui W, Oue N, Aung PP, Matsumura S, Shutoh M, Nakayama H. Molecular pathological prognostic factors of gastric cancer: a review. Gastric Cancer 8(2), 86-94 (2005).

11 Lianos GD, Bali CD, Glantzounis GK, Katsios C, Roukos $\mathrm{DH}$. BMI and lymph node ratio may predict clinical outcomes of gastric cancer. Future Oncol. 10(2), 249-255 (2014). 
12 Washinqton K. 7th edition of the AJCC cancer staging manual: stomach. Ann. Surg. Oncol. 17(12), 3077-3079 (2010).

13 Wu XS, Wu WG, Li ML et al. Impact of being overweight on the surgical outcomes of patients with gastric cancer: a metaanalysis. World J. Gastroenterol. 19(28), 4596-4606 (2013).

14 Ziogas D, Roukos DH. CDH1 testing: can it predict the prophylactic or therapeutic nature of total gastrectomy in hereditary diffuse gastric cancer? Ann. Surg. Oncol. 16(10), 2678-2681 (2009).

15 Lynch HT, Kaurah P, Wirtzfeld D et al. Hereditary diffuse gastric cancer: diagnosis, genetic counseling, and prophylactic total gastrectomy. Cancer 112, 2655-2663 (2008)

16 Kaurah P, MacMillan A, Boyd N et al. Founder and recurrent $\mathrm{CDH} 1$ mutations in families with hereditary diffuse gastric cancer. JAMA 297, 2360-2372 (2007).

17 Kothari N, Almhanna K. Current status of novel agents in advanced gastroesophageal adenocarcinoma. J. Gastrointest. Oncol. 6(1), 60-74 (2015).

18 Aprile G, Giampieri R, Bonotto $\mathrm{M}$ et al. The challenge of targeted therapies for gastric cancer patients: the beginning of a long journey. Expert Opin. Investig. Drugs 23(7), 925-942 (2014).

19 Lianos GD, Vlachos K, Zoras O, Katsios C, Cho WC, Roukos DH. Potential of antibody-drug conjugates and novel therapeutics in breast cancer management. Onco Targets Ther. 7, 491-500 (2014).

20 Wang K, Kan J, Yuen ST et al. Exome sequencing identifies frequent mutation of ARID1A in molecular subtypes of gastric cancer. Nat. Genet. 43, 1219-1223 (2011).

21 Zang ZJ, Cutcutache I, Poon SL et al. Exome sequencing of gastric adenocarcinoma identifies recurrent somatic mutations in cell adhesion and chromatin remodeling genes. Nat. Genet. 44, 570-574 (2012).

22 Wong SS, Kim KM, Ting JC et al. Genomic landscape and genetic heterogeneity in gastric adenocarcinoma revealed by whole-genome sequencing. Nat. Commun. 5, 5477 (2014).

23 Wang K, Yuen ST, Xu J et al. Whole-genome sequencing and comprehensive molecular profiling identify new driver mutations in gastric cancer. Nat. Genet. 46(6), 573-582 (2014). 